\title{
Assessment of the impact of EHR heterogeneity for clinical research through a case study of silent brain infarction
}

\author{
Sunyang Fu', Lester Y. Leung ${ }^{2}$, Anne-Olivia Raulli², David F. Kallmes ${ }^{3}$, Kristin A. Kinsmann ${ }^{3}$, Kristoff B. Nelson²,
} Michael S. Clark ${ }^{3}$, Patrick H. Luetmer ${ }^{3}$, Paul R. Kingsbury', David M. Kent ${ }^{4}$ and Hongfang Liu ${ }^{1 *}$ (D)

\begin{abstract}
Background: The rapid adoption of electronic health records (EHRs) holds great promise for advancing medicine through practice-based knowledge discovery. However, the validity of EHR-based clinical research is questionable due to poor research reproducibility caused by the heterogeneity and complexity of healthcare institutions and EHR systems, the cross-disciplinary nature of the research team, and the lack of standard processes and best practices for conducting EHR-based clinical research.

Method: We developed a data abstraction framework to standardize the process for multi-site EHR-based clinical studies aiming to enhance research reproducibility. The framework was implemented for a multi-site EHR-based research project, the ESPRESSO project, with the goal to identify individuals with silent brain infarctions (SBI) at Tufts Medical Center (TMC) and Mayo Clinic. The heterogeneity of healthcare institutions, EHR systems, documentation, and process variation in case identification was assessed quantitatively and qualitatively.

Result: We discovered a significant variation in the patient populations, neuroimaging reporting, EHR systems, and abstraction processes across the two sites. The prevalence of SBI for patients over age 50 for TMC and Mayo is 7.4 and $12.5 \%$ respectively. There is a variation regarding neuroimaging reporting where TMC are lengthy, standardized and descriptive while Mayo's reports are short and definitive with more textual variations. Furthermore, differences in the EHR system, technology infrastructure, and data collection process were identified.

Conclusion: The implementation of the framework identified the institutional and process variations and the heterogeneity of EHRs across the sites participating in the case study. The experiment demonstrates the necessity to have a standardized process for data abstraction when conducting EHR-based clinical studies.
\end{abstract}

Keywords: Electronic health records, Reproducibility, Clinical research informatics, Data quality, Multi-site studies, Learning health system

\footnotetext{
* Correspondence: liu.hongfang@mayo.edu

'Department of Health Sciences Research, Mayo Clinic, Rochester, MN, USA

Full list of author information is available at the end of the article
}

(c) The Author(s). 2020 Open Access This article is licensed under a Creative Commons Attribution 4.0 International License, which permits use, sharing, adaptation, distribution and reproduction in any medium or format, as long as you give appropriate credit to the original author(s) and the source, provide a link to the Creative Commons licence, and indicate if changes were made. The images or other third party material in this article are included in the article's Creative Commons licence, unless indicated otherwise in a credit line to the material. If material is not included in the article's Creative Commons licence and your intended use is not permitted by statutory regulation or exceeds the permitted use, you will need to obtain permission directly from the copyright holder. To view a copy of this licence, visit http://creativecommons.org/licenses/by/4.0/ The Creative Commons Public Domain Dedication waiver (http://creativecommons.org/publicdomain/zero/1.0/) applies to the data made available in this article, unless otherwise stated in a credit line to the data. 


\section{Background}

The rapid adoption of electronic health records (EHRs) holds great promise for transforming healthcare with EHR enabled continuously learning health systems (LHS), first envisioned by the Institute of Medicine in 2007 [1]. A continuously learning health system can enable efficient and effective care delivery with the ability to discover practice-based knowledge and the seamless integration of clinical research with care practice $[2,3]$. To achieve such a vision, it is critical to have a robust data and informatics infrastructure with the following properties: 1) high-throughput and real-time methods for data extraction and analysis, 2) transparent and reproducible processes to ensure scientific rigor in clinical research, and 3) implementable and generalizable scientific findings $[1,2,4-7]$.

One common approach to practice-based knowledge discovery is chart review, a process of extracting information from patient medical records to answer a specific clinical question $[8,9]$. Traditionally, chart review is performed manually and follows a pre-defined abstraction protocol [10]. Since a significant portion of clinical information is embedded in text, this manual approach can be time-consuming and costly [10-14]. With the implementation of EHRs, chart review can be automated by extracting data from structured fields systematically and leveraging natural language processing (NLP) techniques to extract information from text. Multiple studies have been reported to leverage NLP for extracting information from a diverse range of document types, such as clinical notes, radiology reports, and surgical operative reports [15-17], resulting in an effort reduction of up to $90 \%$ in chart review [18]. The development and evaluation of NLP algorithms for a specific chart review task requires the manual creation of a gold standard clinical corpus, however, there is a lack of standard processes or best practices for creating such a corpus [19, 20].

Meanwhile, reproducibility is crucial in the field of medicine where findings of a single site study must be able to be independently validated at different sites [21-24]. It is very challenging to validate an EHRbased study, due to the heterogeneity and complexity of EHR systems, the challenge of collaboration across diverse research stakeholders (i.e. physician, informatician, statistician, and IT), and the lack of standard processes and best practices for conducting EHRbased studies [19, 20, 25]. The lack of detailed study protocols, such as annotation guidelines and abstraction forms, can cause a study to not be reproducible, even at the same site [26]. For example, Gilbert et al. reviewed research articles published in three emergency medicine journals and discovered that among all studies related to retrospective chart review, only $11 \%$ reported the use of an abstraction form [14].
Challenges faced in leveraging EHR data lie in the voluminous, complex, and dynamic data being generated and maintained in heterogeneous sources. Madigan et al. systematically assessed the variability of 10 different clinical databases and discovered that between 20 to $40 \%$ of observational database studies can alter from statistically significant in one direction to an opposite direction [27]. A research study conducted by Sohn at el, assessing clinical documentation variations across two different EHRs, discovered potential corpus variability (i.e. number of clinical concepts per patient and number of clinical concepts per document are different) in unstructured text [28]. Another challenge found between heterogeneous EHRs is missing and noisy data [29]. Since different EHRs may have different causes underlying their missing data, unintentional bias may be introduced if the issue is ignored or poorly handled [30]. These variations and challenges need to be considered when developing solutions for information extraction and knowledge translation. To facilitate multi-site studies [4], efforts are underway to link EHRs across institutions and to standardize the definition of phenotypes for large-scale studies of disease onset and treatment outcomes in routine clinical care [31-34], however, unstructured data still remains a challenge.

In the clinical NLP community, efforts have been made to standardize corpus development including building and sharing annotated lexical resources, normalizing data elements, and developing an ontology-based web tool [13, 35-37]. However, to the best of our knowledge, there has been little informatics investigation done regarding the impact of using EHRs for clinical research in multiinstitutional settings. Here, we conducted a multi-site EHR-based case study in the ESPRESSO (Effectiveness of Stroke Prevention in Silent Stroke) [38] project involving multiple steps to generate a corpus for the development of complex phenotype algorithms. The heterogeneity of healthcare institutions, EHR systems, documentation, and process variation in case identification was assessed quantitatively and qualitatively.

\section{Methods \\ Data abstraction framework for EHR-based clinical research}

We developed a data abstraction framework to standardize the process for multi-site EHR-based clinical studies aiming to enhance research reproducibility. The development of the proposed framework was designed after review of the existing guidelines and best practices, including Corpus $\mathrm{An}$ notation Schemes [39]; Fundamentals of clinical trials [40]; and Research data management [41]. Figure 1 presents the process of creating annotated corpora for EHR-based clinical research. 


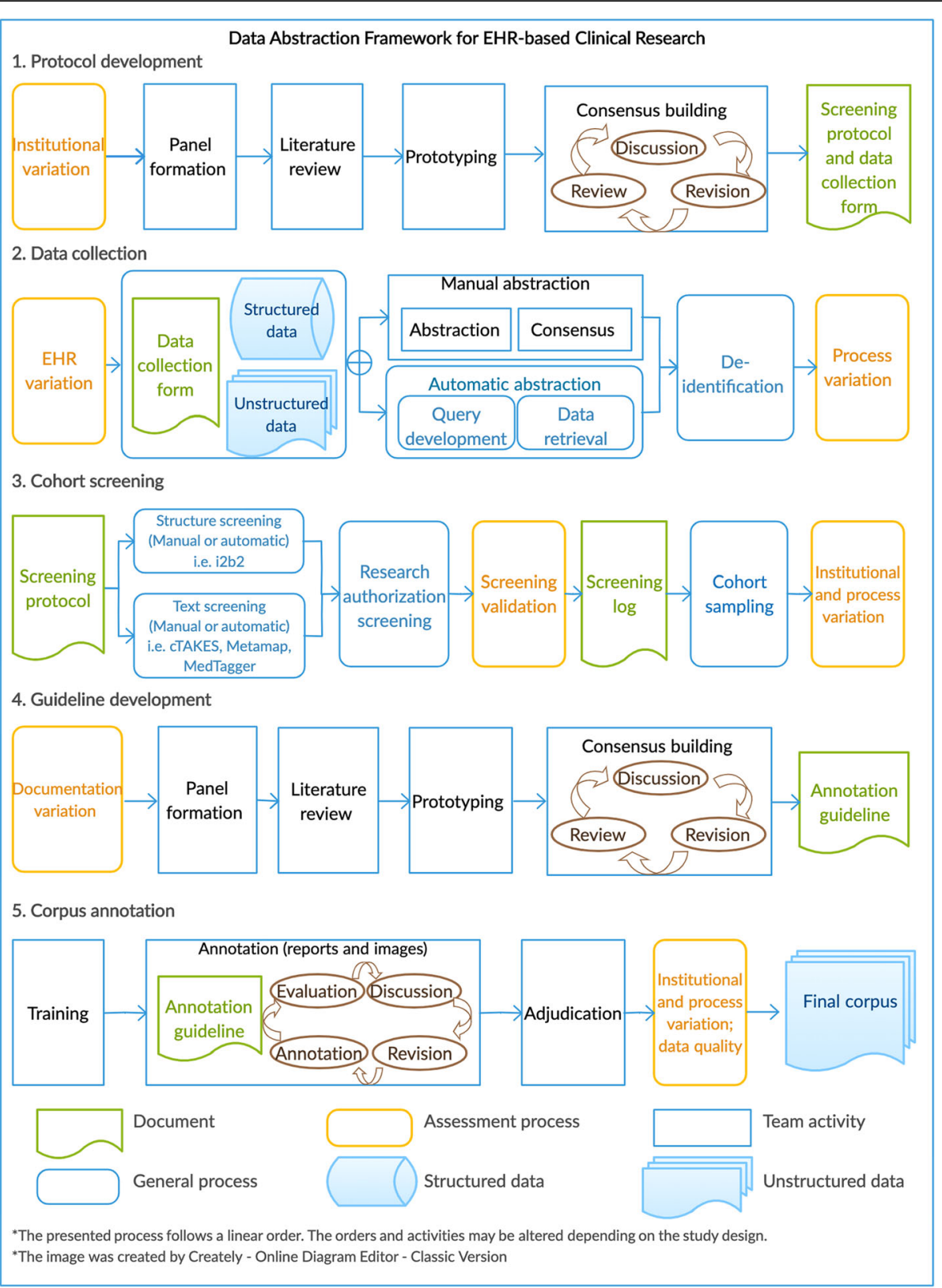

Fig. 1 Data Abstraction Framework for EHR-based Clinical Research

The framework summarizes the linear process of extracting or reviewing information from EHRs and assembling a data set for various research needs. The processes consider important action items and documentation checklist to identify, evaluate and mitigate variations across sites. Depending on the study design, the order of processes and selection of activities can be altered. We considered four types of variations: institutional variation, EHR system variation, documentation variation, and process variation (Fig. 1, yellow boxes). Table 1 summarizes the definitions, potential implication and assessment methodologies of these variations.

\section{A case study - the ESPRESSO study}

This ESPRESSO study is an EHR-based study aiming to estimate the comparative effectiveness of preventive therapies on the risk of future stroke and dementia in patients with incidentally-discovered brain infraction [38, 42]. The study has been approved by the Mayo Clinic and Tufts Medical Center institutional review boards. Mayo Clinic is a tertiary care, nonprofit, academic medical center. Mayo Clinic is a referral center with major campuses in three regions of the U.S. including Rochester, Minnesota; Jacksonville, Florida; and Phoenix/Scottsdale, Arizona as well as Mayo Clinic Health System locations that serve more than 
Table 1 Variation Assessment Table for Data Abstraction

\begin{tabular}{|c|c|c|c|}
\hline Variation Type & Definition & Potential Implication & Example of Assessment Method \\
\hline Institutional variation & $\begin{array}{l}\text { Variation in practice patterns, } \\
\text { outcomes, and patient } \\
\text { sociodemographic characteristics }\end{array}$ & $\begin{array}{l}\text { Inconsistent phenotype } \\
\text { definition; unbalanced } \\
\text { concept distribution }\end{array}$ & $\begin{array}{l}\text { - Compare clinical guideline, protocol, } \\
\text { and definition } \\
\text { - Calculate the number of eligible patients } \\
\text { divided by screening population } \\
\text { - Calculate the ratio of the proportion of the } \\
\text { persons with the disease over the proportion } \\
\text { with the exposure }\end{array}$ \\
\hline EHR system variation & $\begin{array}{l}\text { Variation in data type and format } \\
\text { caused by different EHR system } \\
\text { infrastructure }\end{array}$ & $\begin{array}{l}\text { Inconsistent data type; } \\
\text { different data collection } \\
\text { processes }\end{array}$ & $\begin{array}{l}\text { - Compare data type, document structure, and } \\
\text { metadata } \\
\text { - Conduct a semi-structured interview to obtain } \\
\text { information about the context of use }\end{array}$ \\
\hline Documentation variation & $\begin{array}{l}\text { Variation in reporting schemes } \\
\text { during the processes of generating } \\
\text { clinical narratives }\end{array}$ & Noisy data & $\begin{array}{l}\text { - Compare the cosine similarity between two } \\
\text { documents represented by vectors } \\
\text { - Conduct a sub-language analysis to assess } \\
\text { syntactic variation }\end{array}$ \\
\hline Process variation & $\begin{array}{l}\text { Variation in data collection and } \\
\text { corpus annotation process }\end{array}$ & $\begin{array}{l}\text { Poor data reliability, } \\
\text { validity, and reproducibility }\end{array}$ & $\begin{array}{l}\text { - Calculate the degree of agreement among } \\
\text { abstractors } \\
\text { - Conduct a semi-structured interview to } \\
\text { obtain information about the context of use }\end{array}$ \\
\hline
\end{tabular}

70 communities in Iowa, Wisconsin and Minnesota. The organization attends to nearly 1.2 million patients each year, who come from throughout the United States and abroad. The Saint Mary's (1,265 licensed beds) and Rochester Methodist (794 beds) campuses are two main hospitals located in Rochester, Minnesota. Tufts Medical Center is similarly a tertiary care, nonprofit, academic medical center that is located in Boston, MA and is the principal teaching hospital of the Tufts University School of Medicine. The 415 licensed bed medical center provides comprehensive patient care across a wide variety of disciplines with disease-specific certifications through the Joint Commission as a Comprehensive Stroke Center and transplant center. TMC is the referral center for the WellForce network serving communities throughout Eastern Massachusetts and New England (Maine, New Hampshire, Vermont, Rhode Island). The medical center is actively engaged in clinical research and medical education with ACGME-accredited residencies and fellowships.

Silent brain infarction (SBI) is the presence of one or more brain infarcts, presumed to be due to vascular occlusion, found by neuroimaging in patients without clinical manifestations of stroke [43-45]. It is more common than a stroke and can be detected in $20 \%$ of healthy elderly people [43-45]. Early detection of SBI may prompt efforts to mitigate the risk of stroke by offering preventative treatment plans. In addition to SBI, white matter disease (WMD) or leukoaraiosis is another common finding in neuroimaging of older patients. SBI and WMD are related, but it is unclear whether they result from the same, independent, or synergistic processes [46, 47]. Since SBIs and WMDs are usually incidentally detected, there are no related International Classification of Diseases (ICD) codes in the structured fields of EHRs to facilitate large-scale screening. Instead, the findings are usually recorded in neuroimaging reports, so NLP techniques offer an opportunity to systematically identify SBI and WMD cases in EHRs.

In the study, we demonstrated the process of using EHRs for developing complex phenotypes to identify individuals with incidentally-discovered SBIs and WMDs. The process was assessed by corpus statistics, screening ratio, prevalence ratio, inter-annotator agreement, and qualitative interview.

\section{Methodologic process of using EHRs Protocol development}

A screening protocol was co-developed by the two institutions using procedure codes, diagnosis codes, and problem lists. The protocol included ICD-9 and ICD-10 codes to identify non-incidental clinical events. The codes were expanded with the corresponding descriptions to enable us to perform a text search. The full ICD-9 and ICD-10 codes and key terms are listed in the Additional file 1 . The initial criteria were developed by a vascular neurologist at TMC and were evaluated by two neuroradiologists and one internist. The inclusion criteria were defined as individuals with neuroimaging scans between 2009 and October 2015. The exclusion criteria included patients with clinically-evident stroke, transient ischemic attack (TIA), and dementia any time before or up to 30 days after the imaging exam. TIA was considered an exclusion criterion as TIA is sometimes incorrectly assigned on occasion by clinicians as the diagnosis in the setting of transient neurologic symptoms and positive evidence of brain infarction on neuroimaging. Dementia was an exclusion criterion because of a projected future application of the NLP algorithm in identifying patients for comparative effectiveness studies or clinical trials for which both stroke and dementia 
could be outcomes of interest. The systematic reviews suggested that the U.S. population over 50 years old had a high average prevalence of SBI [44]. By identifying a large cohort of patients with SBIs, age restriction was applied to exclude individuals 50 years of age or younger at the time of the first neuroimaging scan.

\section{Data collection}

At TMC, the data was aggregated and retrieved from three EHR systems: General Electric Logician, eClinicalWorks, and Cerner Soarian. The EHRs in TMC were implemented in 2009 with 1,031,159 unique patient records. At Mayo Clinic, the data was retrieved from the Mayo Unified Data Platform (UDP), an enterprise data warehouse which loads data directly from Mayo EHRs. Mayo EHR was implemented in 1994. Currently, there are 9,855,533 unique patient records. To allow data sharing across the sites, we de-identified the data by applying the de-identification tool DEID [48], a Java-based software that automatically removes protected health information (PHI) in neuroimaging reports with manual verification where an informatician, an abstractor and a statistician manually reviewed all the output from DEID.

\section{Cohort screening}

At Mayo Clinic, an NLP system, MedTagger [49], was utilized to capture mentions from the exclusion list in the clinical notes. As the system has a regular expression component, language variations such as spelling and abbreviations were able to be captured. Structured ICD-9 and ICD-10 codes were obtained by an informatician from the UDP. A clinician and an abstractor manually compared the screened cohort with the EHRs to ensure the validity of the screening algorithm.

At TMC, due to infrastructure limitations, this process was conducted through manual chart review. To ensure reproducibility, we carefully documented each step of the workflow (Additional file 3). Briefly, a vascular neurologist and three research assistants conducted manual chart review in order to determine whether individuals were included or excluded appropriately at each step. This process was performed using a list of free text exclusion criteria associated with the exclusionary ICD-9 and ICD-10 codes. It involved review of the full text of any discharge summaries associated with the encounter during which the neuroimaging scan was obtained in Cerner Soarian, if present, as well as review of the neuroimaging scan indication in the neuroimaging report.

Each site randomly selected 500 eligible reports to form the raw corpus for guideline development and corpus annotation. The cohort consisted of 1400 reports with 400 duplications for double reading. Among the total 400 double-read reports, 5 reports were removed because of invalid scan types. The remaining 395 reports were comprised of 207 from Mayo and 188 from TMC.

\section{Guideline development}

A baseline guideline was created by a vascular neurologist based on domain knowledge and published literature. To develop the annotation guideline, 40 reports pooled from the two institutions were annotated by two neuroradiologists and one neurologist using the baseline guideline. Inter-annotator agreement (IAA) was calculated and a consensus was organized to finalize the guideline, which included task definitions, annotation instructions, annotation concepts, and examples. The full guideline is provided in the Additional file 2.

\section{Corpus annotation}

The annotation processes consist of two tasks: neuroimaging report annotation and neuroimage interpretation. Neuroimaging report annotation is the process of reading and extracting SBI and WMD related sentences or concepts from text documents. Neuroimage interpretation is the process of identifying SBIs or WMDs from $\mathrm{CT}$ or MRI images. Figure 2 provides an example of two tasks.

Neuroimaging report annotation The purpose of the annotation task was to annotate the findings of SBI and WMD lesions in both the body (Findings) and summary (Impression and Assessment) sections of neuroimaging reports. The annotation was organized into two iterations. The first iteration extended from the finalization of the process guideline until the midpoint when half of the reports were annotated. The goal of the first iteration was to identify new problems that were not captured in the sample data. After the first iteration, all problematic cases were reviewed by the two senior clinicians, and the guidelines were updated. The second iteration of annotation then commenced using the updated guidelines. Several consensus meetings were organized to resolve all disagreements after the annotation process was completed. All conflicting cases were adjudicated by the two senior clinicians. All of the issues encountered during the process were documented.

The annotation team was formed with members from both institutions. Two third-year residents from Mayo and two first-year residents from TMC performed the annotation. The experts for quality control were two senior radiologists from Mayo and one senior internist and one vascular neurologist from TMC. We used Multi-document Annotation Environment (MAE) [50], a Java-based natural language annotation software package, to conduct the annotation.

Prior to annotation, training was conducted for all four annotators including one online video session and two 


\section{Severe chronic microvascular degenerative change. periventricular deep white matter most likely related to small vessel ischemic disease.}

\section{Old lacunar infarct in the right thalamus. Moderate parenchymal atrophy. No intracranial restricted diffusion or mass. Small amount of fluid within the mastoid air cells.}

\section{Task 1- Neuroimaging report annotation}
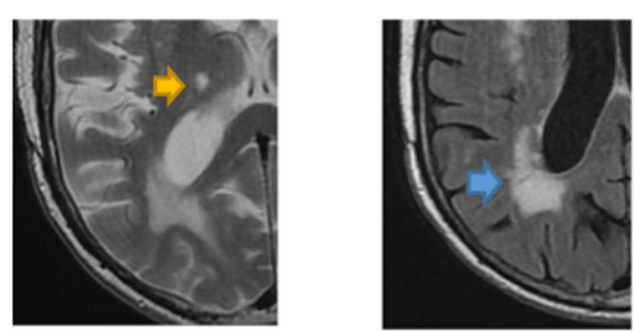

Blue arrow: WMD modified Manolio grading scale of 6 Yellow arrow: chronic right
thalamic infarct, $>3 \mathrm{~mm}$-bright on $\mathbb{T} 2$, low on $\mathbb{T} 1$

\section{Task 2 - Neuroimage interpretation}

Fig. 2 Example of neuroimaging report annotation (left) and neuroimage interpretation (right) for SBI (yellow) and WMD (blue)

on-site training sessions. The online video provided demonstrations and instructions on how to download, install, and use the annotation software. The on-site training conducted by two neuroradiologists contained initial annotation guideline walkthroughs, case studies, and practice annotations. The same clinicians supervised the subsequent annotation process.

Neuroimage interpretation To assess the validity of the corpus, we obtained a balanced sample of images with and without SBI from the annotated neuroimaging reports. From each site, 81 neuroimages were deidentified and reformatted to remove institution-specific information and then pooled together for the sample group. We invited four attending neuroradiologists, two from each site, to read grade the imaging exams. Each exam was graded twice by two neuroradiologists independently. The image reading process followed the proposed best practices including guideline development, image extraction form, training, and consensus building. The level of agreements between the research grade reading of the neuroimages and the corresponding annotation of the reports was calculated.

\section{Assessment of heterogeneity}

The screening ratio was calculated on the post screened cohort. Cohen's kappa [51] and F-measure [52] were adopted to measure the IAA during the annotation and image reading processes. Corpus statistics were used to measure the variations in clinical documentation across institutions. The analysis compared corpus length, number of SBI and WMD concepts, number of documents with SBI and WMD concepts, and distribution of SBI related concept mentions. Document similarity was calculated by comparing the cosine similarity between two vectors created by term frequencyinverse document frequency (tf-idf), where each corpus was represented by a normalized tf-idf vector [28]. Age-specific prevalence of SBI and WMD were calculated and compared with the literature. To analyze the cohort characteristics between Mayo and TMC, Student's t-test was performed for continuous variables. Comparison of categorical variables was calculated with frequency tables with Fisher's exact test.

Qualitative assessments were conducted to evaluate the abstraction process and an assessment protocol was created to facilitate the post abstraction interview. The protocol was designed to focus on three main areas: 1) evaluation of the abstraction process, 2) language patterns in the reports, and 3) abstraction techniques. Four back-to-back interviews were conducted with the four abstractors following the guidelines of Contextual Interview (CI) suggested by Rapid Contextual Design [53]. Each interview was conducted by an informatician and lasted approximately $30 \mathrm{~min}$. Questions and issues raised by each annotator during the two iterations of annotation were collected and qualitatively assessed. The data were then classified into six categories: data, modifier, medical concept, annotation rules, linguistic, and other.

\section{Results}

\section{Corpus annotation}

\section{Neuroimaging report annotation}

The average inter-annotator agreements across 207 Mayo reports and 188 TMC reports on SBI and WMD were 0.87 and 0.98 in kappa score and 0.98 and 0.99 in F-measure, respectively. Overall, both Mayo and TMC annotators achieved high inter-annotator agreements. 


\section{Neuroimage interpretation}

The average inter-annotator agreement among four neuroradiologists was 0.66 in kappa score and 0.83 in Fmeasure. The average agreement between neuroimaging interpretation and corpus annotation was 0.68 in kappa score and 0.84 in F-measure. The result suggested high corpus validity outcomes.

\section{Assessment of heterogeneity Institutional variation}

The process of screening eligible neuroimaging reports across two institutions was variant. At Mayo, 262,061 reports were obtained from Mayo EHR based on the CPT inclusion criteria. 4015 reports were randomly sampled for cohort screening. 749 were eligible for annotation after applying the ICD exclusion criteria (structured and unstructured). At TMC, 63,419 reports were obtained from TMC EHR based on CPT inclusion criteria. 12,092 reports remained after applying the ICD exclusion criteria (structured). 1000 reports were randomly selected for text screening, a method of identifying eligible patients using NLP techniques to extract eligibility criteria from patient clinical notes. 773 reports were eligible for annotation. Among the total 1522 eligible (Mayo 749, TMC 773) neuroimaging reports, 1000 (Mayo 500, TMC 500) reports were randomly selected.

The prevalence of SBI and WMD for Mayo and TMC patients at age of 50, 60, 70 and 80 is listed in Table 2. Despite the variation, the results were consistent with the published literature, between 10 and 20\% [43-45], and the number increased with age in both computed tomography (CT) and magnetic resonance imaging (MRI) as anticipated.

The average age of Mayo and TMC patients 65 and 66 , respectively. The number of female patients in the Mayo and TMC cohort were 243 and 274, respectively. We found a moderate variation in the presence of SBI and WMD and a high variation in the WMD grading. A significant variation in the missing documentation of WMD grading between Mayo and TMC was found $(p=$ 0.0024). Table 3 summarizes the cohort characteristics across two institutions.

\section{EHR system variation}

There was a high variation in the EHR system vendors, the number of EHR systems per site, and the extract, transform, and load (ETL) processes for the different EHR systems between Mayo and TMC. At TMC, the data was obtained directly from three EHR systems: General Electric Logician, eClinicalWorks, and Cerner Soarian. The data retrieval process involved difference abstraction processes due to the different interface design and data transfer capabilities. At Mayo Clinic, there was an ETL process to aggregate the data from Mayo EHRs to the enterprise data warehouse. Since data could be linked and transferred through direct queries, the abstraction process was less variant.

\section{Documentation variation}

There was variation between Mayo and TMC in expressing SBI and WMD in neuroimaging reports. Corpus statistics identified the three most frequent expressions of negated infarction in neuroimaging reports (Table 4). In the TMC reports, "no acute territorial infarction" is a common phrase to describe negated SBI concepts. This expression was never discovered in Mayo reports. When describing the grade measure for WMDs, definitive expressions such as "mild", "moderate" and "severe" were used by Mayo physicians. On the other hand, TMC physicians used more descriptive expressions in describing the grade measure for WMDs. In regards to documentation styles, TMC used a template-based reporting method whereas Mayo did not adopt any reporting schemas. The average numbers of tokens per document on Mayo and TMC reports were 217 and 368, respectively. The corpus similarity between TMC and Mayo Clinic radiology reports was 0.82 and suggested a potential moderate-to-high semantic similarity. Overall, Mayo's reports are definitive and varied, whereas TMC reports are lengthy, standardized and descriptive.

\section{Process variation}

The process map of the ESPRESSO data abstraction is illustrated in Fig. 3 - Part I. The map provides an overview of the relationship and interaction between people and technology in the context of the data abstraction process. The analysis suggested that the variations of EHR systems and technology infrastructures between the two sites have resulted in differences in the number of processing steps, experts, and duration (Fig. 3 - Part II).

Table 2 The prevalence of SBI and WMD for Mayo and TMC patients at age of 50, 60, 70 and 80

\begin{tabular}{|c|c|c|c|c|c|c|c|c|}
\hline \multirow[b]{2}{*}{ Age } & \multicolumn{2}{|c|}{ CT Scan (\%) - SBI } & \multicolumn{2}{|c|}{ MRI Scan (\%) - SBI } & \multicolumn{2}{|c|}{ CT Scan (\%) - WMD } & \multicolumn{2}{|c|}{ MRI Scan (\%) - WMD } \\
\hline & Mayo & TMC & Mayo & TMC & Mayo & TMC & Mayo & TMC \\
\hline$>=50$ & 12.5 & 7.4 & 11.3 & 7.7 & 28.7 & 55.0 & 69.2 & 51.7 \\
\hline$>=60$ & 16.0 & 9.4 & 14.0 & 9.7 & 35.1 & 65.9 & 75.3 & 60.2 \\
\hline$>=70$ & 23.5 & 11.4 & 20.2 & 12.2 & 47.1 & 80.7 & 84.6 & 65.3 \\
\hline$>=80$ & 26.3 & 18.4 & 26.5 & 20.8 & 52.6 & 94.7 & 85.3 & 66.7 \\
\hline
\end{tabular}


Table 3 Analysis of Cohort Characteristics Between Mayo and TMC

\begin{tabular}{|c|c|c|c|}
\hline Variables & Mayo $(n=500)$ & TMC $(n=500)$ & $p$ Value \\
\hline Age (mean) & $65(+-10.6)$ & $66(+-9.7)$ & 0.1197 \\
\hline Gender (female) & $243(48.6)$ & $274(54.8)$ & 0.0576 \\
\hline SBI & $57(11.4)$ & $38(7.6)$ & 0.0516 \\
\hline \multicolumn{4}{|l|}{ Acuity } \\
\hline Acuity/subacute & $6(1.2)$ & $6(1.2)$ & 1.0000 \\
\hline Chronic & $44(8.8)$ & $29(5.8)$ & 0.0882 \\
\hline Non-specified & $7(1.4)$ & $3(0.6)$ & 0.3407 \\
\hline \multicolumn{4}{|l|}{ Location } \\
\hline Lacunar/subcortical & $27(5.4)$ & $10(2.0)$ & 0.0065 \\
\hline Cortical/juxtacortical & $9(1.8)$ & $13(2.6)$ & 0.5188 \\
\hline Both & $0(0)$ & $3(0.6)$ & 0.2492 \\
\hline Non-specified & $21(4.2)$ & $12(2.4)$ & 0.1558 \\
\hline WMD & $291(58.2)$ & $264(52.8)$ & 0.9800 \\
\hline \multicolumn{4}{|l|}{ WMD grading } \\
\hline Mild & $191(38.2)$ & $154(30.8)$ & 0.0165 \\
\hline Mild/moderate & $21(4.2)$ & $0(0.0)$ & $7.6963 \mathrm{e}-7$ \\
\hline Moderate & $42(8.4)$ & $45(9.0)$ & 0.8226 \\
\hline Moderate/severe & $2(0.4)$ & $0(0)$ & 0.4995 \\
\hline Severe & $8(1.6)$ & $11(2.2)$ & 0.6443 \\
\hline No mention of quantification & $27(5.4)$ & $54(10.8)$ & 0.0024 \\
\hline
\end{tabular}

Definition of abbreviations: $\mathrm{Cl}$ confidence interval, OR odds ratio

\section{Recommended practices for EHR-based data abstraction} Throughout this case study, we encountered many challenges during the data abstraction process. Here we summarize some lessons learned and provide a few empirical recommendations to promote the best practices of using EHRs for clinical research.

\section{Institutional variation}

It is inevitable to encounter variabilities across different institutions. Being aware of the degree of variation can help estimate biases and prevent inaccurate study conclusions. Thus, it is always helpful to apply informatics techniques to capture and assess the variation to ensure transparent and informed EHR-based clinical research.

\section{Documentation plan and checklist}

A comprehensive documentation plan for a study allows interventions aimed at process replication and error prevention to be designed into the data abstraction. The plan should explicitly mention what, where, and when to document experimental elements such as protocols, guidelines, codes, operations manuals, and process workflows. Ensuring adequate time is devoted to documentation is critical in order to prevent details from being

Table 4 Example of Language Variation between Two Data Sources

\begin{tabular}{|c|c|}
\hline Mayo - Non-SBI & TMC - Non-SBI \\
\hline $\begin{array}{l}\text { - No restricted diffusion. } \\
\text { - No focal masses, focal atrophy, or foci of } \\
\text { restricted water diffusion. } \\
\text { - No evidence for acute ischemia on the } \\
\text { diffusion weighted images. }\end{array}$ & $\begin{array}{l}\text { - There is no acute territorial infarct. } \\
\text { - No acute territorial infarct. } \\
\text { - There is no decreased diffusion to indicate an acute infarct. }\end{array}$ \\
\hline Mayo - WMD & TMC - WMD \\
\hline $\begin{array}{l}\text { - Mild leukoaraiosis } \\
\text { - Minimal leukoaraiosis } \\
\text { - Moderate leukoaraiosis }\end{array}$ & $\begin{array}{l}\text { - There are scattered foci of hypodensity in the subcortical and periventricular white } \\
\text { matter, a non-specific finding but likely reflecting the sequela of chronic microangiopathy } \\
\text { - Areas of white matter hypodensity are a non-specific finding but may represent the sequela } \\
\text { of chronic microangiopathy } \\
\text { - There are multiple foci of t2 flair hyperintensity in the periventricular, deep and subcortical } \\
\text { white matter, a non-specific finding but likely reflecting the sequela of chronic microangiopathy }\end{array}$ \\
\hline
\end{tabular}




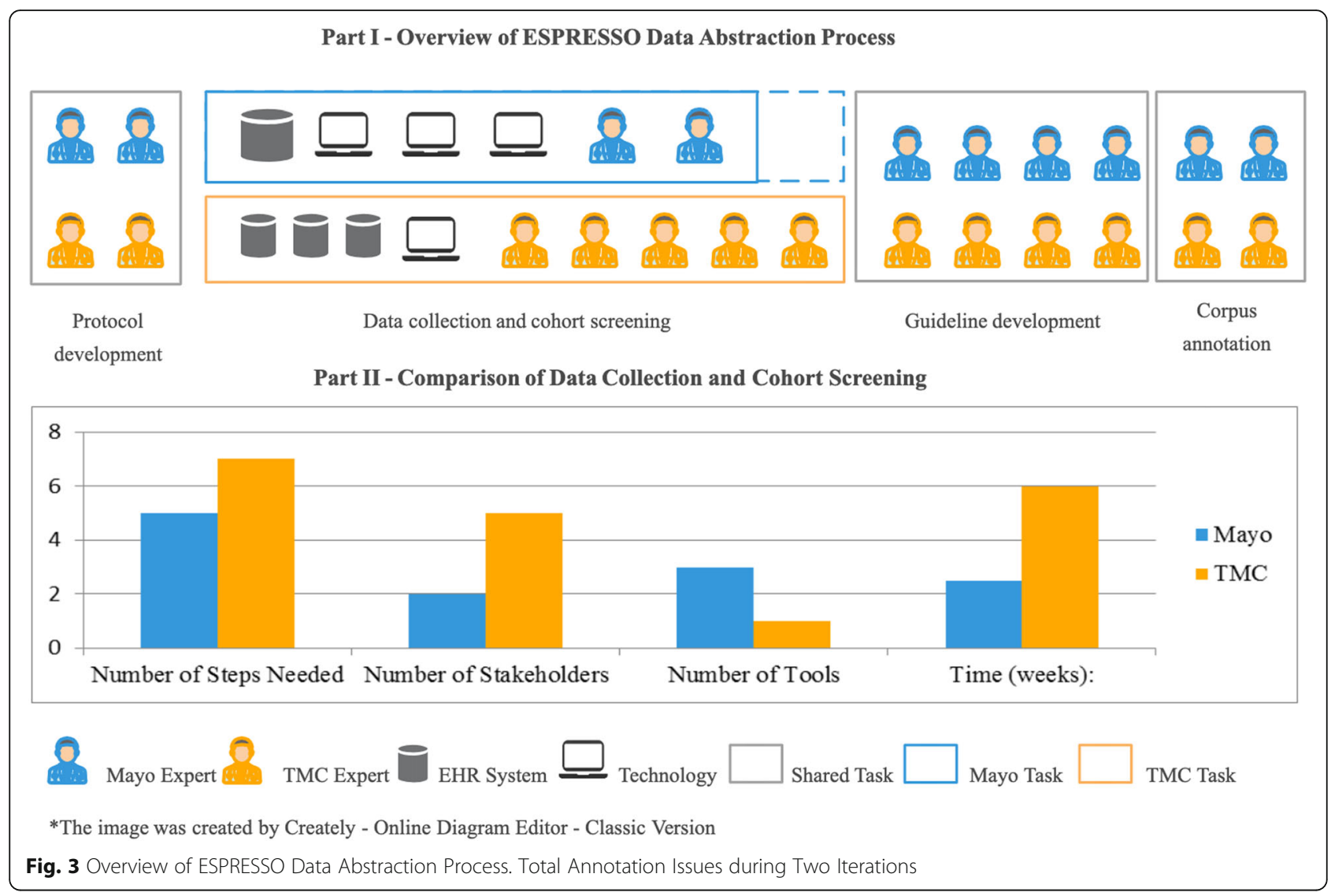

overlooked or omitted. A documentation checklist ensures important study details are documented. Examples of important metadata elements are data identifier (i.e. document id, document date, and patient clinic number), cohort definition (i.e. inclusion and exclusion criteria), steward, and description of the data (when the data is created, moved, modified, or filtered). During data abstraction, process logs, tools, data definitions, and methodologies need to be carefully analyzed and explicitly stated.

\section{Concept definitions and protocol co-development}

To ensure data validity, the variables of the study should be strictly defined. Standardized terminology codes, such as ICD, SNOMED-CT, CPT-4, or RxNorm are useful for describing observable medical characteristics. Protocol codevelopment and consensus building helped reduce institutional and process variance in our study (Figs. 1 and 3). Particularly, having a well-represented expert panel (from all sites) for developing and evaluating inclusion and exclusion criteria and annotation guidelines helped the creation of high-quality protocol documents.

\section{Abstraction and annotation training}

Proper training and education can help reduce process inconsistency and increase transparency, especially for a cross disciplinary team. When the training sessions were applied, a shared understanding of rigorous experimental design, research standards, and objective evaluation of data was ensured. Some example training activities included discussing the overall study goal, going through the contents of the annotation guideline and definitions of interest, and practicing using the annotation tool (i.e. allowing people to work on a sample of 5-10 notes).

\section{Process iteration and consensus building}

A consensus reaching process is an iterative and dynamic process for building agreement on any potential issues and disagreements. A consensus meeting should be organized when developing screening protocols and annotation guidelines. Routine discussions ensure guidelines and protocols are scientifically valid and robust.

\section{Adoption of appropriate informatics tools}

Successfully leveraging informatics techniques can improve process efficiency, data quality, and reproducibility. For example, automatic data retrieval techniques (such as application programming interface and structured query language) and cohort screening tools (such as i2b2 [54]) can enable a high-throughput data abstraction process. Using annotation tools ensures a standardized and reproducible annotation process. It is more 
important to choose an appropriate informatics solution than an advanced solution. In the study, we chose a light and standalone version of annotation software over an advanced web-based tool due to its high feasibility and efficiency. In situations that require extensive validation for processes, such as de-identification, human validations are needed after applying the informatics tools.

\section{Discussion}

We conducted a multi-site EHR-based case study in the implementation of the ESPRESSO project to assess the impact of EHR heterogeneity for clinical research. The case study discovered significant variation regarding patient population, neuroimaging reporting, EHR systems, and abstraction processes. Despite the variation, the evaluation of the final corpus yielded high reliability and validity.

The assessment through the ESPRESSO discovered a high variation in the reported prevalence of SBIs between Mayo and TMC. There are two potential reasons for the low prevalence of SBIs in TMC. First, the two locations have different patient sociodemographic characteristics at the two locations. Although both Mayo and TMC are referral centers, Mayo may have a larger proportion of patients who are referred from distant locations whereas TMC may have predominantly local and regional referrals. Second, low SBI prevalence may be due to the different documentation priorities during the routine practice. For further investigation, a qualitative assessment was utilized to learn how clinicians report neuroimaging interpretations. Based on the analysis of cohort characteristics between Mayo and TMC (Table 3) and the post abstraction interview, we discovered a portion of SBIs were under-documented by TMC neuroradiologists due to their historical perceptions of potentially low clinical significance for SBIs. For example, the descriptions about the clinical utility of reporting on small and presumably asymptomatic brain lesions that could represent infarcts were very uncertain. Compared with TMC, the wording describing SBIs on the Mayo reports was more definitive.

Although the average kappa score on the Mayo reports was lower than the TMC reports, the score still reflected an exceptionally high agreement between all annotators. We believe this was achieved by a well-designed process. During guideline development, we found that variation could be reduced by adding an instruction manual to the guidelines. Due to the large number of reports that were assigned to each resident, the de-identified reports were equally distributed to individuals as a "take home" assignment. The instruction manual helped to guide annotation activities, such as suggesting the number of reports that needed to be annotated per day. One of the most commonly raised issues was the lack of precise modifier definitions for WMD. To reduce the abstraction variation caused by different interpretation of modifiers, we created a normalization mapping schema. For example, the level of grading for WMDs was explicitly defined to be mild, mild/moderate, moderate, moderate/ severe, and severe.

The qualitative assessment of the annotation process (Fig. 1 - process 5 - box 2) identified that medical concepts (i.e. mention of SBI and WMD) and modifiers (i.e. acuity and location) were the primary issues during the first iteration of annotation. Additional training was offered to address the primary issues experienced during the first iteration of annotation and thus, decreased the occurrence of issues during the second iteration (Fig. 3). All four annotators noted that with the combination of training and comprehensive annotation guidelines, annotation time was shortened, effort redundancy was reduced, and annotation consistency was improved.

\section{Limitations and future work}

Since the study was conducted on two sites with one case scenario, the generalizability of the process is limited by the scope of the study. Our next step is to expand our investigation on pragmatic clinical trials by incorporating more sites and case scenarios. Furthermore, we plan to develop a standardized process framework for EHR-based clinical research to ensure the validity, reliability, reproducibility and transparency of research findings.

\section{Conclusion}

We conducted a case study based on the ESPRESSO project identified the institutional and process variations and the heterogeneity of EHRs across sites. Our experiment demonstrates the necessity to have a standardized process for the use of EHRs for clinical studies.

\section{Supplementary information}

Supplementary information accompanies this paper at https://doi.org/10. 1186/s12911-020-1072-9.

Additional file 1: Supplemental Appendix 1. Screening Protocol.

Additional file 2: Supplemental Appendix 2. Annotation Guideline.

Additional file 3: Supplemental Appendix 3. Process Documentation TMC Manual Screening.

\section{Abbreviations}

EHR: Electronic Health Records; TMC: Tufts Medical Center; LHS: Learning Health System; NLP: Natural Language Processing; ESPRESSO: Effectiveness of Stroke Prevention in Silent Stroke; WMD: White Matter Disease; SBI: Silent Brain Infraction; ICD: International Classification of Diseases; PHI: Protected Health Information; IAA: Inter-annotator Agreement; MAE: Multi-document Annotation Environment; TF-IDF: Term Frequency-Inverse Document Frequency; MRI: Magnetic Resonance Imaging; CT: Computed Tomography; ETL: Extract, transform, and load 


\section{Acknowledgements}

We gratefully acknowledge Katelyn Cordie, Sungrim Moon, Andrew Wen, and Luke A Carlson for editorial support and Donna M Ihrke for case validation.

\begin{abstract}
Authors' contributions
$H L$ conceived the study and design. LYL and SF acquired the data and implemented the algorithms. LYL created drafted initial protocol and guideline. AOR, KAK, KN, MSC, DFK, PHL performed the annotation. LYL, DMK, DFK, SF, and PHL participated guideline creation and supervised annotation. SF performed the analysis and wrote the manuscript. PRK and HL conducted editorial work. All authors participated in interpretation of the data and contributed to manuscript revisions. The author(s) read and approved the final manuscript.
\end{abstract}

\section{Funding}

This work was made possible by National Institute of Health $(\mathrm{NIH})$ grant number R01NS102233 and U01TR002062-01.

\section{Availability of data and materials}

The datasets used and/or analyzed during the current study are available from the corresponding author on reasonable request.

\section{Ethics approval and consent to participate}

Our study used existing records to conduct a retrospective study. The study and a waiver of informed consent were approved by Mayo Clinic and Tufts Medical Center institutional review boards (Approval \#17-006674, Board/ Committee: Minimal Risk).

\section{Consent for publication}

Not applicable.

\section{Competing interests}

The authors declare that they have no competing interests.

\section{Author details}

'Department of Health Sciences Research, Mayo Clinic, Rochester, MN, USA. ${ }^{2}$ Department of Neurology, Tufts Medical Center, Boston, MA, USA. ${ }^{3}$ Department of Radiology, Mayo Clinic, Rochester, MN, USA. Institute for Clinical Research and Health Policy Studies, Tufts Medical Center, Boston, MA, USA

\section{Received: 8 April 2019 Accepted: 12 March 2020}

Published online: 30 March 2020

\section{References}

1. Friedman CP, Wong AK, Blumenthal D. Achieving a nationwide learning health system. Sci Transl Med. 2010;2(57):57cm29.

2. Gelijns AC, Gabriel SE. Looking beyond translation--integrating clinical research with medical practice. N Engl J Med. 2012;366(18):1659-61.

3. Milstein A. Code red and blue--safely limiting health care's GDP footprint. N Engl J Med. 2013;368(1):1-3.

4. Richesson RL, Horvath MM, Rusincovitch SA. Clinical research informatics and electronic health record data. Yearb Med Inform. 2014;9:215-23.

5. Kaggal VC, Elayavilli RK, Mehrabi S, Pankratz JJ, Sohn S, Wang Y, Li D, Rastegar MM, Murphy SP, Ross JL, et al. Toward a learning health-care system - knowledge delivery at the point of care empowered by big data and NLP. Biomed Inform Insights. 2016;8(Suppl 1):13-22.

6. Curcin V. Embedding data provenance into the learning health system to facilitate reproducible research. Learning Health Systems. 2016;1 (2):e10019.

7. Wen A, Fu S, Moon S, El Wazir M, Rosenbaum A, Kaggal VC, Liu S, Sohn S, Liu H, JJnDM F. Desiderata for delivering NLP to accelerate healthcare Al advancement and a Mayo Clinic NLP-as-a-service implementation. NPJ Digit Med. 2019:2(1):1-7..

8. Frankovich J, Longhurst CA, Sutherland SM. Evidence-based medicine in the EMR era. N Engl J Med. 2011;365(19):1758-9.

9. Gearing RE, Mian IA, Barber J, Ickowicz A. A methodology for conducting retrospective chart review research in child and adolescent psychiatry. J Can Acad Child Adolesc Psychiatry. 2006;15(3):126-34.

10. Vassar M, Holzmann M. The retrospective chart review: important methodological considerations. J Educ Eval Health Prof. 2013;10:12.
11. Xu H, Jiang M, Oetjens M, Bowton EA, Ramirez AH, Jeff JM, Basford MA, Pulley JM, Cowan JD, Wang X. Facilitating pharmacogenetic studies using electronic health records and natural-language processing: a case study of warfarin. J Am Med Inform Assoc. 2011:18(4):387-91.

12. Grishman R, Huttunen $S$, Yangarber R. Information extraction for enhanced access to disease outbreak reports. J Biomed Inform. 2002;35(4):236-46.

13. South BR, Shen S, Jones M, Garvin J, Samore MH, Chapman WW, Gundlapall AV. Developing a manually annotated clinical document corpus to identify phenotypic information for inflammatory bowel disease. BMC Bioinformatics. 2009;10(Suppl 9):S12.

14. Gilbert EH, Lowenstein SR, Koziol-McLain J, Barta DC, Steiner J. Chart reviews in emergency medicine research: where are the methods? Ann Emerg Med. 1996:27(3):305-8.

15. Wu ST, Sohn S, Ravikumar K, Wagholikar K, Jonnalagadda SR, Liu H, Juhn YJ. Automated chart review for asthma cohort identification using natural language processing: an exploratory study. Ann Allergy Asthma Immunol. 2013:111(5):364-9.

16. Dresser MV, Feingold L, Rosenkranz SL, Coltin KL. Clinical quality measurement. Comparing chart review and automated methodologies. Med Care. 1997;35(6):539-52.

17. Melton GB, Hripcsak G. Automated detection of adverse events using natural language processing of discharge summaries. J Am Med Inform Assoc. 2005;12(4):448-57.

18. Carrell DS, Halgrim S, Tran DT, Buist DS, Chubak J, Chapman WW, Savova G. Using natural language processing to improve efficiency of manual chart abstraction in research: the case of breast cancer recurrence. Am J Epidemiol. 2014;179(6):749-58.

19. Cohen KB, Xia J, Roeder C, Hunter LE. Reproducibility in natural language processing: a case study of two R libraries for mining PubMed/MEDLINE. LREC Int Conf Lang Resour Eval. 2016:2016(W23):6-12.

20. Branco A. Reliability and meta-reliability of language resources: ready to initiate the integrity debate? In: 12th Workshop on Treebanks and Linguistic Theories: December 13-14, 2013 2013; Sofia, Bulgaria; 2013.

21. Baker D, Lidster K, Sottomayor A, Amor S. Reproducibility: research-reporting standards fall short. Nature. 2012;492(7427):41.

22. Johnson KE, Kamineni A, Fuller S, Olmstead D, Wernli KJ. How the provenance of electronic health record data matters for research: a case example using system mapping. EGEMS (Wash DC). 2014:2(1):1058.

23. Karczewski KJ, Tatonetti NP, Manrai AK, Patel CJ, Titus Brown C, loannidis JPA. Methods to ensure the reproducibility of biomedical research. Pac Symp Biocomput. 2017;22:117-9.

24. Anderson WP. Reproducibility: stamp out shabby research conduct. Nature. 2015;519(7542):158.

25. Zozus MN, Richesson RL, Walden A, Tenenbaum JD, Hammond WE. Research reproducibility in longitudinal multi-center studies using data from electronic health records. AMIA Jt Summits on Transl. 2016;2016: 279-85.

26. Manrai AK, Patel CJ, Gehlenborg N, Tatonetti NP, loannidis JP, Kohane IS. Methods to enhance the reproducibility of precision medicine. Pac Symp Biocomput. 2016;21:180-2

27. Madigan D, Ryan PB, Schuemie M, Stang PE, Overhage JM, Hartzema AG, Suchard MA, DuMouchel W, Berlin JA. Evaluating the impact of database heterogeneity on observational study results. Am J Epidemiol. 2013;178(4): $645-51$.

28. Sohn S, Wang Y, Wi Cl, Krusemark EA, Ryu E, Ali MH, Juhn YJ, Liu H. Clinical documentation variations and NLP system portability: a case study in asthma birth cohorts across institutions. J Am Med Inform Assoc. 2018;25(3): 353-9.

29. Kharrazi HH, Wang C, Scharfstein DO. Prospective EHR-based clinical trials: The challenge of missing data. J Gen Intern Med. 2014;29(7): 976-8.

30. Wells BJ, Chagin KM, Nowacki AS, Kattan MWJE. Strategies for handling missing data in electronic health record derived data. EGEMS (Wash DC) 2013;1(3):1035.

31. Weber GM, Murphy SN, McMurry AJ, Macfadden D, Nigrin DJ, Churchill S, Kohane IS. The shared Health Research information network (SHRINE): a prototype federated query tool for clinical data repositories. J Am Med Inform Assoc. 2009:16(5):624-30.

32. Selby JV, Beal AC, Frank L. The Patient-Centered Outcomes Research Institute (PCORI) national priorities for research and initial research agenda. JAMA. 2012;307(15):1583-4. 
33. Consortium PCP, Daugherty SE, Wahba S, Fleurence R. Patient-powered research networks: building capacity for conducting patient-centered clinical outcomes research. J Am Med Inform Assoc. 2014;21(4):583-6.

34. Hripcsak G, Duke JD, Shah NH, Reich CG, Huser V, Schuemie MJ, Suchard MA, Park RW, Wong IC, Rijnbeek PR, et al. Observational health data sciences and informatics (OHDSI): opportunities for observational researchers. Stud Health Technol Inform. 2015;216:574-8.

35. Savova GK, Chapman WW, Zheng J, Crowley RS. Anaphoric relations in the clinical narrative: corpus creation. J Am Med Inform Assoc. 2011;18(4):459-65.

36. Albright D, Lanfranchi A, Fredriksen A, Styler WF, Warner C, Hwang JD, Choi JD, Dligach D, Nielsen RD, Martin J, et al. Towards comprehensive syntactic and semantic annotations of the clinical narrative. J Am Med Inform Assoc. 2013;20(5):922-30.

37. Scuba W, Tharp M, Mowery D, Tseytlin E, Liu Y, Drews FA, Chapman WW. Knowledge author: facilitating user-driven, domain content development to support clinical information extraction. J Biomed Semantics. 2016;7(1):42.

38. Leung LY, Han PK, Lundquist C, Weinstein G, Thaler DE, Kent D. Clinicians' perspectives on incidentally discovered silent brain infarcts-a qualitative study. PLoS One. 2018;13(3):e0194971.

39. Leech G. Corpus annotation schemes. Literary Linguist Comput. 1993;8(4): 275-81.

40. Friedman LM, Furberg C, DeMets DL. Fundamentals of clinical trials: springer; 1998.

41. Strasser C. Research data management. National Information Standards Organization; 2015

42. Fu S, Leung LY, Wang Y, Raulli A-O, Kallmes DF, Kinsman KA, Nelson KB, Clark MS, Luetmer PH. Kingsbury PRJJmi: Natural Language Processing for the Identification of Silent Brain Infarcts From Neuroimaging Reports. 2019; 7(2):e12109.

43. Vermeer SE, Longstreth WT Jr, Koudstaal PJ. Silent brain infarcts: a systematic review. Lancet Neurol. 2007;6(7):611-9.

44. Fanning JP, Wong AA, Fraser JF. The epidemiology of silent brain infarction: a systematic review of population-based cohorts. BMC Med. 2014;12:119.

45. Fanning JP, Wesley AJ, Wong AA, Fraser JF. Emerging spectra of silent brain infarction. Stroke. 2014;45(11):3461-71.

46. Conklin J, Silver FL, Mikulis DJ, Mandell DM. Are acute infarcts the cause of leukoaraiosis? Brain mapping for 16 consecutive weeks. Ann Neurol. 2014; 76(6):899-904.

47. Chen Y, Wang A, Tang J, Wei D, Li P, Chen K, Wang Y, Zhang Z. Association of white matter integrity and cognitive functions in patients with subcortical silent lacunar infarcts. Stroke. 2015;46(4):1123-6.

48. Aberdeen J, Bayer S, Yeniterzi R, Wellner B, Clark C, Hanauer D, Malin B, Hirschman L. The MITRE identification scrubber toolkit: design, training, and assessment. Int J Med Inform. 2010;79(12):849-59.

49. Liu H, Bielinski SJ, Sohn S, Murphy S, Wagholikar KB, Jonnalagadda SR, Ravikumar KE, Wu ST, Kullo IJ, Chute CG. An information extraction framework for cohort identification using electronic health records. AMIA Jt Summits Transl Sci Proc. 2013;2013:149-53.

50. Rim K. Mae2: Portable annotation tool for general natural language use. In: 12th Joint ACL-ISO Workshop on Interoperable Semantic Annotation, vol. 2016; 2016.

51. Cohen J. A coefficient of agreement for nominal scales. Educ Psychol Meas. 1960;20(1):37-46.

52. Sasaki Y. The truth of the F-measure. Teach Tutor Mater. 2007;1(5):1-5.

53. Holtzblatt KWJ, Wood S. Rapid contextual design: a how-to guide to key techniques for user-centered design: Elsevier; 2004

54. Murphy SN, Weber G, Mendis M, Gainer V, Chueh HC, Churchill S, IJJotAMIA K. Serving the enterprise and beyond with informatics for integrating biology and the bedside (i2b2). J Am Med Inform Assoc. 2010;17(2):124-30.

\section{Publisher's Note}

Springer Nature remains neutral with regard to jurisdictional claims in published maps and institutional affiliations.

Ready to submit your research? Choose BMC and benefit from:

- fast, convenient online submission

- thorough peer review by experienced researchers in your field

- rapid publication on acceptance

- support for research data, including large and complex data types

- gold Open Access which fosters wider collaboration and increased citations

- maximum visibility for your research: over $100 \mathrm{M}$ website views per year

At BMC, research is always in progress.

Learn more biomedcentral.com/submissions 